\title{
The ANDES Deep Underground Laboratory
}

\author{
Xavier Bertou* \\ CNEA/CONICET, Centro Atómico Bariloche, Argentina \\ E-mail: bertoulcab.cnea.gov.ar
}

The Agua Negra Deep Experiment Site (ANDES) is a planned deep underground laboratory in the soon-to-be-built Agua Negra tunnel between Argentina and Chile. It will offer about $4000 \mathrm{~m}^{2}$, $70000 \mathrm{~m}^{3}$ underground space for frontier science experiments in basic science, with a strong focus on Dark Matter search and Neutrino physics. A specific large section of the laboratory will be dedicated to geosciences, and smaller cavities will be available for multidisciplinary studies. Being located in the deepest portion of the tunnel, with $1700 \mathrm{~m}$ of overburden, it will be both a deep and large laboratory, with a direct horizontal road access. Being in the southern hemisphere, it may play a crucial role in Dark Matter and Neutrino physics and aims to be a key player in the exploration of the Dark Side of the Universe.

2nd World Summit: Exploring the Dark Side of the Universe 25-29 June, 2018 - EDSU2018

University of Antilles, Pointe-à-Pitre, Guadeloupe, France

${ }^{*}$ Speaker. 


\section{Introduction}

Deep underground laboratories are key actors in the physics of weakly interactive particles such as the Neutrino or possibly Dark Matter candidates. They have diversified in the last decades to also host a significant combination of multidisciplinary experiments, based on low background radioactivity measurements, biology studies, or geoscience observations.

Figure 1 shows the location of current and future deep underground laboratories around the world. All the currently operating laboratories are in the northern hemisphere. ANDES will have a unique location allowing it to be a key player in Dark Matter search through the study of modulation effects (both annual where comparison with the northern hemisphere can exclude atmospheric seasonal effects and sign a genuine extra-terrestrial signal modulation[2], and daily in the case of strong energy losses or self-interacting Dark Matter[3, 4]), in some study of neutrino physics (such as MSW propagation effects for supernova neutrinos or geoneutrinos[5]), and for geoscience, given the strong geoactivity of the region.

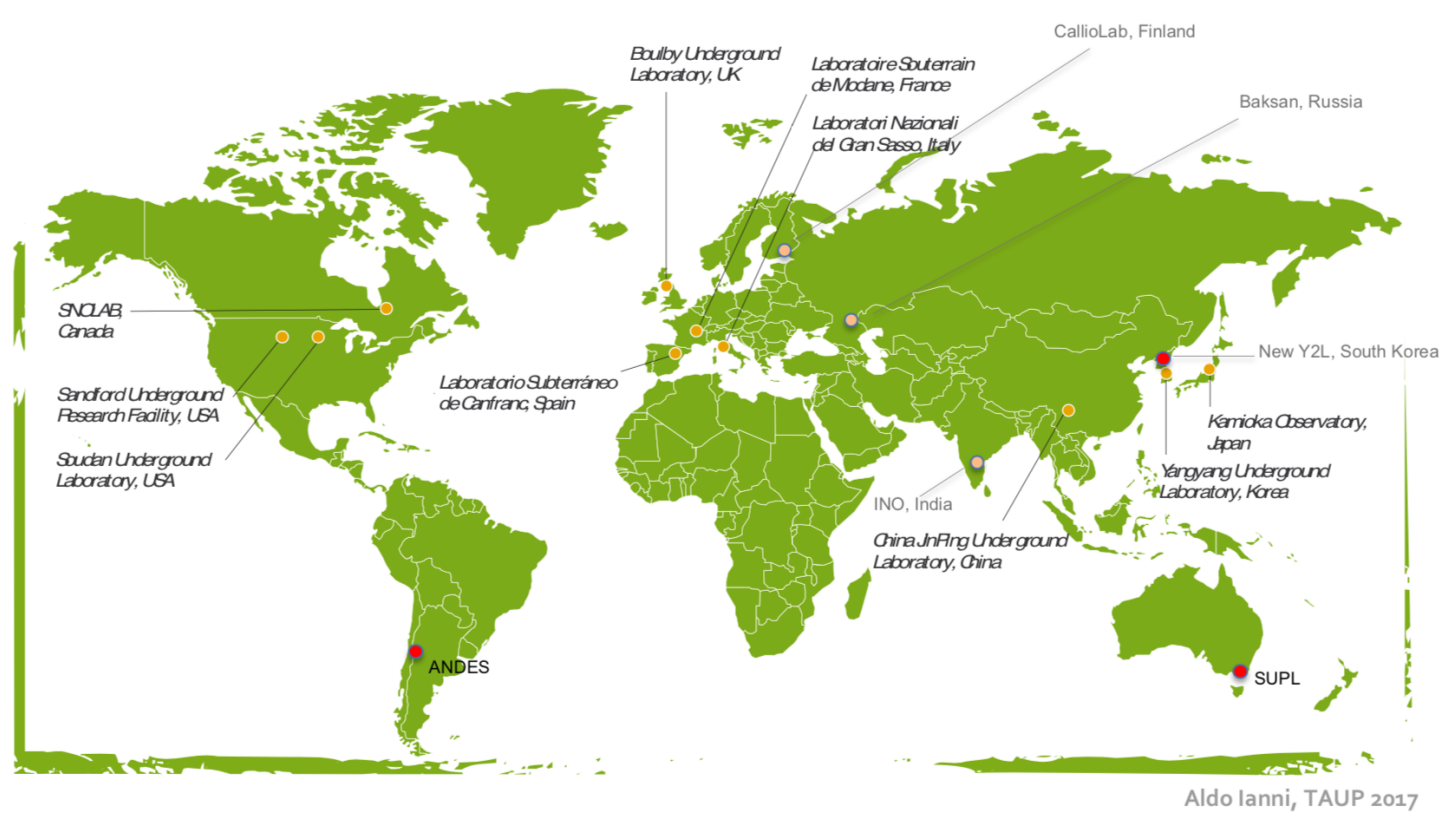

Figure 1: Location of current and future deep underground laboratories around the world[1]. See text for details.

\section{ANDES in the Agua Negra tunnel}

The Agua Negra tunnel is composed of two parallel $14 \mathrm{~km}$ long road tunnels at the frontier between Argentina and Chile (in the San Juan province in Argentina and the Coquimbo region in Chile). It is located at high altitude with the Argentine portal at $4085 \mathrm{~m}$ of altitude while the Chilean portal is at $3620 \mathrm{~m}$. It aims at increasing the connectivity of Argentina and Brazil to China through the pacific ocean taking benefit of the Chilean infrastructure. It will be a binational area, which construction has been approved by the two countries, is supervised by the EBITAN (Entidad BInacional Túnel Agua Negra) binational technical and political entity, voted by both congresses. 
The financing for the $1.5 \mathrm{~B} \$$ civil work is provided by the Interamerican Development Bank (IDB). $280 \mathrm{M} \$$ out of the $1.5 \mathrm{~B} \$$ have already been approved and the tender process is expected to finish in 2019, with construction to start soon afterwards. The construction process is expected to last 9 years.

ANDES has been proposed to the EBITAN twice, in 2012 and 2017 in its $\mathrm{X}^{\text {th }}$ and $\mathrm{XXXV}^{\text {th }}$ meetings, and has been approved for inclusion into the civil work. A New Conceptual Design (NEC) for the laboratory has been elaborated by the Lombardi civil work company in 2016, and the basic engineering and tender documentation (IBA+DTL) has been initiated in August 2018. It is expected to have all the necessary documentation by end of 2018 in order to include ANDES into the tunnel civil work. The total cost of the civil work for ANDES was estimated in the NEC to be of the order of $40 \mathrm{M} \$$.

The layout for the laboratory has proposed in the NEC and in the current version of the preliminary IBA+DTL at the time of writing (November 2018) is shown in figure 2. A definitive layout is expected by the end of the year, including a geoscience section that was not present in the NEC. Other modifications from the NEC are related to construction and safety issues.

The main experiment spaces are foreseen to be a main hall of $21 \mathrm{~m}$ width, $23 \mathrm{~m}$ high and $50 \mathrm{~m}$ long, that will host sizable experiments together with a large pit of $30 \mathrm{~m}$ of diameter and $30 \mathrm{~m}$ of height. A secondary cavern of $16 \mathrm{~m}$ by $14 \mathrm{~m}$ and $40 \mathrm{~m}$ long will host smaller experiments. Specific caverns are foreseen for different activities such as clean room, low background measurement, biology laboratory, workshop, offices, services, and more. A long $200 \mathrm{~m}$ tunnel ends ANDES and is dedicated to geosciences.

Detailed studies of muon and neutron flux at the laboratory altitude were performed, together with radioactivity measurements of rocks from the geological study of the area, showing no particular issue for the construction of the laboratory [6]. A more detailed study of the underground muon flux expected was conducted to define the possible implantation area of the laboratory. A wide area of $500 \mathrm{~m}$ long between the frontier (deepest point) and the tunnel ventilation cavern (500 $\mathrm{m}$ to the Argentine side) was identified where the muon flux is at its lowest and constant within $10 \%$.

In addition to the underground installation, two support laboratories are foreseen, one close-by in Argentina, focused on daily operation, and a further but larger one in Chile (in La Serena). Both will be nexus to the local universities and research centers, and host a large visitor center.

The construction of ANDES will be concomitant to the construction of the tunnel, and the opening of the laboratory should also occur together with the opening of the tunnel, in 2028.

\section{ANDES Operation}

ANDES is foreseen as an international laboratory. Its scientific programme aims at excellence in basic science, with strong focus on Dark Matter search and Neutrino physics, multidisciplinary studies, with a dedicated biology laboratories, low radiation measurement area, and geoscience with a large dedicated section.

ANDES is currently coordinated from within a specific unit created in January 2014 within the Latin American Center for Physics (CLAF), a class 2 UNESCO institute recognized by most latinamerican countries. It is foreseen to have a wider audience and to be an international initiative governed by a council with member and observer states, similar in structure to SESAME[7], for 


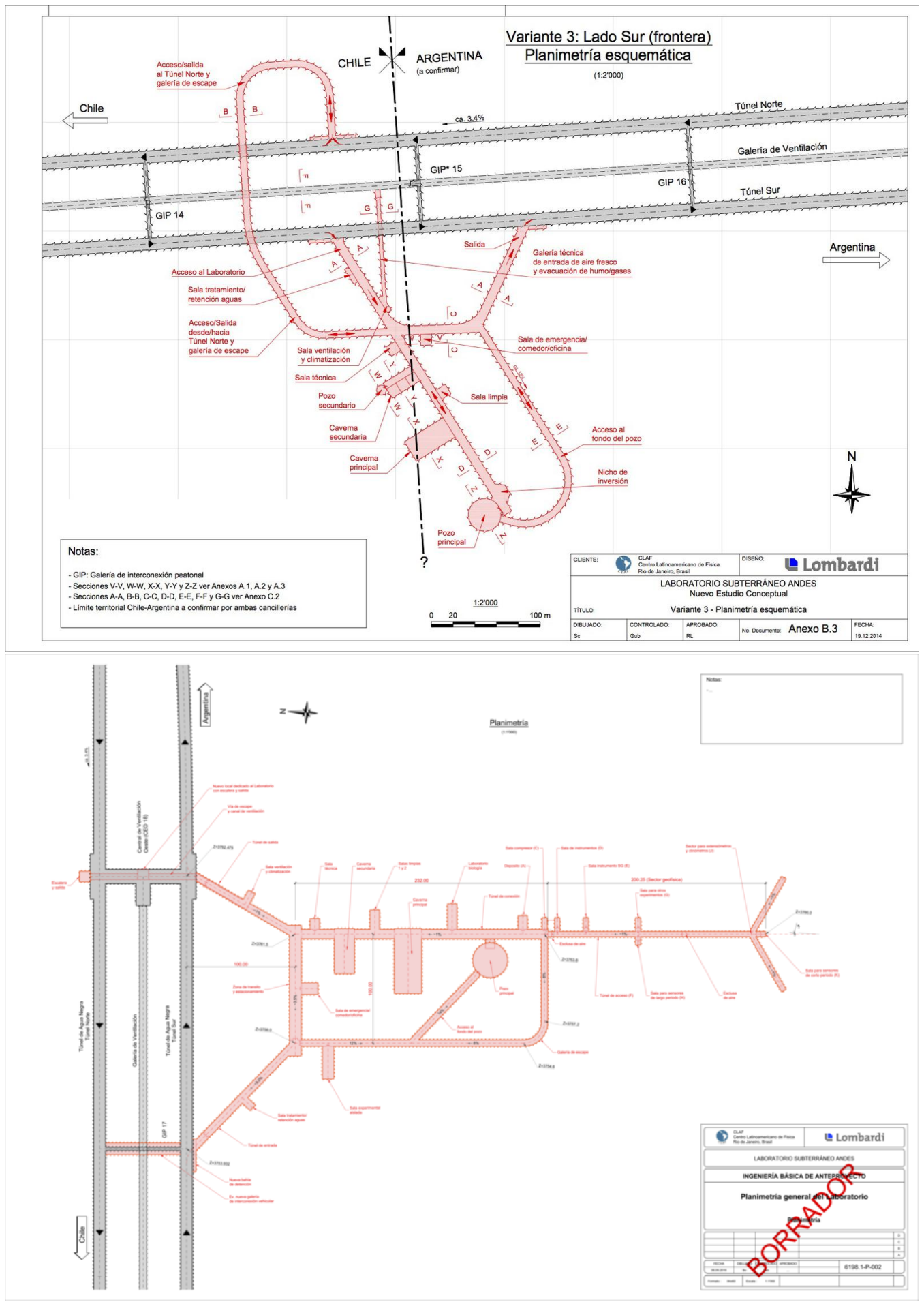

Figure 2: Layout for the ANDES laboratory in the NEC (2016, top) and in the preliminary IBA+DTL (2018, bottom). The latest layout is simplified from the earlier version to reduce costs, and includes a specific section for the geoscience (the final part further away from the tunnel). 
example. While many latinamerican countries are expected to be part of the ANDES initiative, it is foreseen to have countries outside the region both as members and observers. No regional limitation is expected.

More specific white papers and statuses are being worked on. They are expected to be available in 2019 and posted on the ANDES laboratory repository [8].

\section{Conclusions}

The exploration of the Dark Side of the Universe is an extremely active process as was demonstrated by the many efforts presented at the $2^{\text {nd }}$ World Summit. Underground laboratories are key actors in this exploration and the presence of a large and deep site in the southern hemisphere would be a key asset for the community. The ANDES Deep Underground Laboratory initiative fulfills all the requirements to bring to the community a world class laboratory in the southern hemisphere. It should play a leading role in the future of this exploration.

\section{Acknowledgements}

The author would like to thank the conference organizers for the invitation and the opportunity to contribute to a such a successful event.

\section{References}

[1] A. Ianni, TAUP 2017 review talk

[2] R. Bernabei et al, J. Phys.: Conf. Ser. 1056012005 (2018)

[3] C. Kouvaris, I. Shoemaker, Phys. Rev. D 90, 095011 (2014)

[4] R. Foot, S. Vagnozzi, Phys. Lett. B 748, 61âĂŞ66 (2015)

[5] P. A. N. Machado, T. Mühlbeier, H. Nunokawa, R. Zukanovich Funchal, Phys. Rev. D 86, 125001 (2012)

[6] X. Bertou, Eur. Phys. J. Plus 127: 104 (2012)

[7] http://www.sesame.org.jo/

[8] http://andeslab.org/ 